\title{
Development of Novel adsorbent for Industrial waste water treatment
}

\author{
Hassaan M. Shehata ${ }^{1}$, Dalia A. Ali ${ }^{1}$, Islam M. Al-Akraa ${ }^{1}$, Hany A. Elazab ${ }^{1,2^{*}}$, Hoda A. Elsawy ${ }^{1}$ \\ ${ }^{1}$ Department of Chemical Engineering, The British University in Egypt, El-Shorouk City, Cairo, Egypt. \\ ${ }^{2}$ Nanotechnology Research Centre (NTRC), the British University in Egypt (BUE), El-Sherouk City, Suez Desert \\ Road, Cairo, 11837, Egypt. \\ *Elazabha@vcu.edu
}

\begin{abstract}
The main objective of this research project is to perform some experimental procedures to inform the ability of the novel adsorbent used in this project. The chemical modification of the adsorbent is the chemical oxidation of pure powder chitosan using some specific redox pairs which are potassium dichromate and sodium bisulphite. After preparing the modified chitosan, there are some parameters that must be discussed during the adsorption process. The first parameter is the time parameter, showing experimentally the optimum time for adsorption under certain conditions by collecting values of absorbance and concentration of thymol blue adsorbed after the process from the spectrophotometer showing that the optimum time is 120 minutes. While for the optimum $\mathrm{pH}$ experiment, it is shown that optimum $\mathrm{pH}$ is 4 , $0.5 \% \mathrm{v} / \mathrm{v}$ is optimum concentration and 0.05 grams is the optimum dose for the adsorbent. Then after computing these conditions and values of absorbance, some calculations and classifications are done to show the reaction kinetics and isotherm classification of the process. By performing some equations using mathematical rules it is provided that the reaction is not a first order reaction, showing by calculations and graphs that the reaction is a second order reaction. Also, to know the classification of the process other mathematical relations are done calculating slope and $\mathrm{R}^{2}$ to show whether the process reaction is a Langumir or Frendulish, showing that the reaction process is a Frendulish process having a greater value of $R^{2}$.
\end{abstract}

Key words: Dye removal, Metal oxides, Catalyst, Wastewater treatment, Novel adsorbent.

\section{INTRODUCTION}

In our world there are many types of pollutants which can affect the environment, where environmental pollutants are considered to be the greatest pollution problems that human beings are facing in this world.[1-18] There are three types of pollution which are considered the main types of pollution. These types are classified into three basic types which are; water, air and soil.[19-35] Among these types of pollutants water pollution is caused by the usage of excess pesticides and fertilizers in agriculture, industrial discharge and waste water emitted in textile industries which contains a large amount of dyes that are the mostly pollutant for water.[36-42] Dyes which are found in waste water effluent are always difficult to be separated from water streams as they are complex molecular structures that make dyes able to be stable in water toward light and resistant to biodegradation. Synthetic dyes were discovered in the $19^{\text {th }}$ century, while the usage of natural dyes has been started from about 5000 years, but synthetic dyes took the lead in textile industries which has covered up the use of natural dye. Synthetic dyes are involved in many industries and can be produced largely in such industries like; leather, fabrics, food, cosmetics, electroplating and distillation.[43-55]

These synthetic dyes can be classified into different classes such as; basic, acidic, direct, disperse, pigment and reactive dyes and also other classes which are not mentioned. This classification reflects the macroscopic behaviour and prevailing functionalities. Studies proved that more than 700000 tons of dye are produced annually. Also, an estimation proved that about $10-15 \%$ of dyes are gone astray in wastewater mainly in the manufacturing and application processes. In this work, finding a solution for the treatment of wastewater contaminated mainly by dyes is our big issue, where a lot of surveys and searches proved that cationic and anionic dyes are involved largely in many industries such like paper, silk, wool and cosmetics industries [56-60], So many investigations have been done to find a good and a low cost solution to remove theses dye from wastewater effluents, where adsorption was found to be the most effective way to remove these dye from wastewater effluents due to its low cost and easy way of operation in addition to the effectiveness of removing dyes from wastewater effluents [61-63]. So, chitosan was chosen to be the novel adsorbent which can remove cationic and anionic dyes from these effluents but of course some modifications must be held on this adsorbent to 
give the highest effect of adsorption and adsorb as much as it can [64,65]. Oxidation of chitosan was the chosen modification for chitosan since studies proved that it was the most effective modification on chitosan through studying the parameter affecting the adsorption after oxidation takes place [20,30, 61-66].

As an additional investigation to see how adsorption work with dyes, not only the chemical and physical methods are used, there is also biological methods can be used in order to remove dyes from wastewaters. Here in this research the adsorption process using a novel modified adsorbent will be applied in order to remove a certain dye from industrial wastewater.

\section{EXPERIMENTAL}

\subsection{Chitosan Oxidation}

The oxidation process of chitosan is applied using some chemical reagents which help in proceeding the oxidation quickly. First of all, a small amount of pure powder chitosan is prepared to be dissolved in an acetic acid solution which is diluted under certain conditions like temperature and stirring rate, where the temperature must be adjusted on $40{ }^{\circ} \mathrm{C}$ and 700 rounds per minute for four hours long. After dissolving the chitosan powder two reagent are added by sufficient amounts to the solution in a three necked flask and kept also at temperature $40^{\circ} \mathrm{C}$ for 4.5 hours long to be quenched after the oxidation process by sodium hydroxide solution and an ice bath to obtain the hydrogel chitosan before passing it through some processes like filtration, washing by distillate water and drying in an oven at temperature $40^{\circ} \mathrm{C}$ till it has a constant weight.

\subsection{Time Optimization}

After obtaining an amount of oxidized chitosan, firstly the effect of time is studied. As mentioned above that there are some depending parameters that affect the adsorption capacity [7]. In this paper, reaching to the optimum conditions is the desired work to know the optimum conditions of oxidized chitosan to get the best adsorption capacity. Thymol blue is used as the dye desired to be removed. So, a certain concentration of thymol blue is prepared which is $0.5 \% \mathrm{v} / \mathrm{v}$. After adjusting the $\mathrm{pH}$ of the six beakers, the weighted six samples of chitosan are poured in six conical flasks with the solution in each one after adjustment. Before placing the conical flasks in the shaker to begin the adsorption process, the flasks are labelled, where each one is labelled referring to the lengths of time that each flask will take in the shaker starting from 5 minutes reaching to 2 hours $\{5,10,30,60,90,120\}$ minutes with stirring rate 250 rounds per minute.

Six samples are placed in the shaker to begin the adsorption process and when it is time to each sample to be removed, filtration takes place. After finishing the hours shaking, these six samples are taken to spectrophotometer to measure the adsorption capacity through knowing the concentration of dye before and after shaking.

\section{$2.3 \mathrm{pH}$ Optimization}

The optimum time experiment showed the optimum time needed for the modified chitosan to adsorb as much as it can [8]. In the previous experiment all conditions were constant except the variation of time intervals, where $\mathrm{pH}$ and concentration of thymol blue were 4 and $0.5 \%$ respectively and mass of adsorbent is constant too which is 0.05 grams. In this experiment the same conditions are prepared but with a wide change in values of $\mathrm{pH}$ of each beaker to conduct the optimum $\mathrm{pH}$ that the adsorbent can evaluate highest adsorption capacity. The six beakers filled with thymol blue solution are then taken to adjust the $\mathrm{pH}$. Six beakers are divided into two parts equally, providing three acidic beakers and three basic beakers. Three acidic beakers are adjusted using hydrochloric acid obtaining three different values of $\mathrm{pH}$ which are $\{2,4,6\}$, knowing that the $\mathrm{pH}$ of thymol blue solution without any adjustments is 6 so, no drops of $\mathrm{HCl}$ are needed to obtain $\mathrm{pH}$ of 6 . On the other hand, basic beakers are adjusted using sodium hydroxide drops, obtaining different values of $\mathrm{pH}$ which are $\{8,10$, 12\}. After adjusting the $\mathrm{pH}$, these beakers are taken to be poured in six conical flasks labelled with its $\mathrm{pH}$ value to proceed the adsorption process by adding the six weighed samples of modified chitosan to thymol blue solution. The adsorption will take two hours shaking with stirring rate 250 rounds per minute, knowing from the previous experiment the optimum time for adsorption.

\subsection{Concentration Optimization}

In this experiment the optimum concentration of thymol blue is observed by preparing 6 different samples of thymol blue each with its concentration. The original sample of thymol blue which is $0.5 \% \mathrm{v} / \mathrm{v}$ is taken to be diluted obtaining other five concentrations, to show the adsorption capacity of oxidized chitosan with different concentrations of thymol blue. These different concentrations are prepared the same way that $0.5 \% \mathrm{v} / \mathrm{v}$ thymol blue is prepared but using the $0.5 \% \mathrm{v} / \mathrm{v}$ thymol blue and distilled water. As by some calculations it was found that each sample needs a certain amount from distilled water and certain amount from diluted thymol blue, where these concentrations having values of $\{0.4,0.3,0.2,0.1,0.05\}$.

Before mixing the oxidized chitosan with the different concentrations a $\mathrm{pH}$ adjustment is required, where the optimum $\mathrm{pH}$ for chitosan adsorption is 4 . These samples are adjusted using $0.1 \mathrm{M}$ hydrochloric acid. After adjustment, sample in each beaker is poured to its conical flask labelled by its concentration then mixing the weighed oxidized chitosan to start adsorption on the shaker leaving the reaction for two hours with stirring rate 250 rounds per minute.

\subsection{Dose of Oxidized Chitosan Optimization}

In such an experiment different masses of oxidized chitosan are used with constant time, $\mathrm{pH}$ and concentration, to show 
the optimum mass/dose of oxidized chitosan needed to give the best adsorption capacity with these parameters. Five different samples of oxidized chitosan are prepared having different masses where these values are $\{0.05,0.04,0.03$, $0.02,0.01\}$ grams. As done before in the previous experiments, five beakers are prepared each containing $25 \mathrm{ml}$ of $0.5 \% \mathrm{v} / \mathrm{v}$ thymol blue. These beakers are taken to the $\mathrm{pH}$ meter for adjustment, hydrochloric acid of $0.1 \mathrm{M}$ is used for adjusting these samples reaching them to $\mathrm{pH}$ of 4 . Then each sample is poured to the conical flask having its mass labeled mixing the weighed samples of oxidized chitosan with thymol blue, where each mass is filled in its flask. These flasks are taken to the shaker to proceed the adsorption reaction keeping the process for two hours shaking with stirring rate 250 rounds per minute.

\section{RESULTS AND DISCUSSION}

\subsection{Standard Curve}

As shown in the standard curve table, that at different concentrations of thymol blue, different values of absorbance in $(\mathrm{nm})$ are given by the spectrophotometer, as the concentration of thymol blue increase the values of absorbance increase giving the shown values in table 2. So, the least concentration which is $0.1 \% \mathrm{v} / \mathrm{v}$ has the least absorbance value which is $0.011 \mathrm{~nm}$, while the largest concentration which is $0.5 \% \mathrm{v} / \mathrm{v}$ gives an absorbance value of $0.0588 \mathrm{~nm}$.

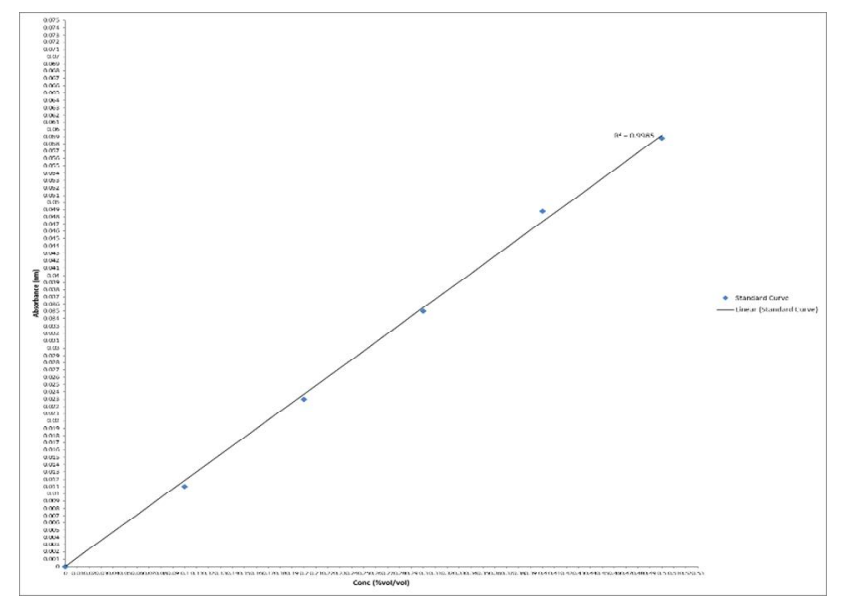

Figure 1: The relation between thymol blue concentration and absorbance

This figure shows the relation between the concentration of thymol blue and the absorbance value given from the spectrophotometer, fitting the line on the best fit of dots to give a straight line, where concentration of thymol blue is on $\mathrm{x}$-axis and absorbance on $\mathrm{y}$-axis. Also, $\mathrm{R}^{2}$ is calculated showing a promising result having a value of 0.9985 . This relation was plotted to make it easy for each experiment to get the concentration of thymol blue after adsorption to know how much thymol blue are adsorbed on the surface of oxidized chitosan, knowing the optimum condition in each experiment, where the least value of thymol blue concentration after adsorption gives the optimum condition of the experiment whatever any experiment.

\subsection{Optimum Time}

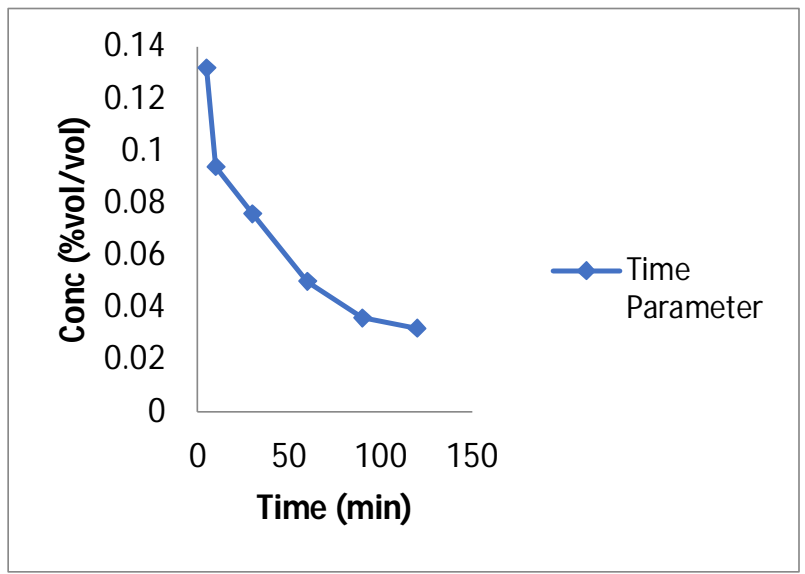

Figure 2: The relation between thymol blue concentration and time of adsorption

Figure 2 shows the relation between the concentration of thymol blue after adsorption and time for adsorption, whereas mentioned above that concentration of thymol blue decreases with time, the more time it spends in shaking the more concentration it loses.

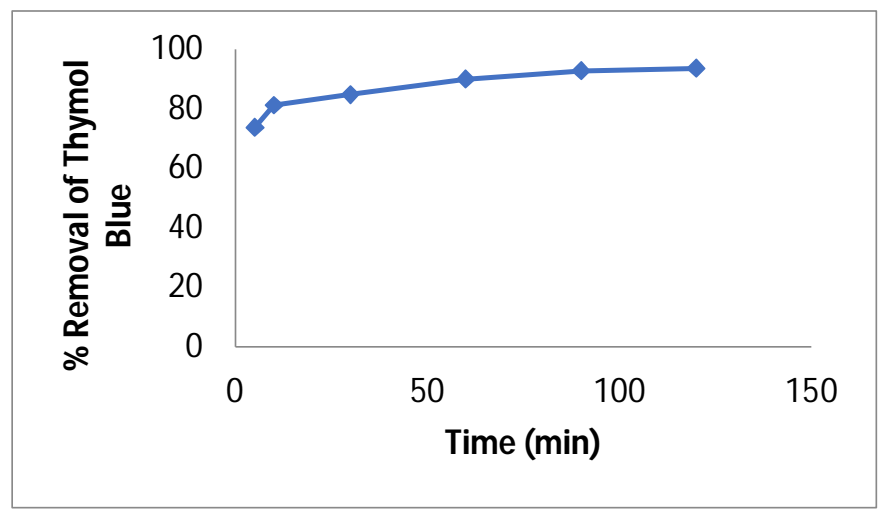

Figure 3: The relation between thymol blue removal percentage and time of adsorption

Figure 3 shows the relation between $\%$ of removal and time is opposite to that of figure (2). Where, percent of removal increases with time as shown in graph, where \% of removal is 93.6 after two hours of shaking.

\section{3 pH Parameter}

Figure 4 shows the unstable conditions of samples having $\mathrm{pH}$ greater than 4 , whereas the $\mathrm{pH}$ increases from 2 to 4 , percent of removal increases showing the best value of removal, but as the increase to be more than 4 the curve start to drop showing unstable curves and unstable percentage of removal till 
reaching the most basic medium which is 12 having the lowest percent of removal.

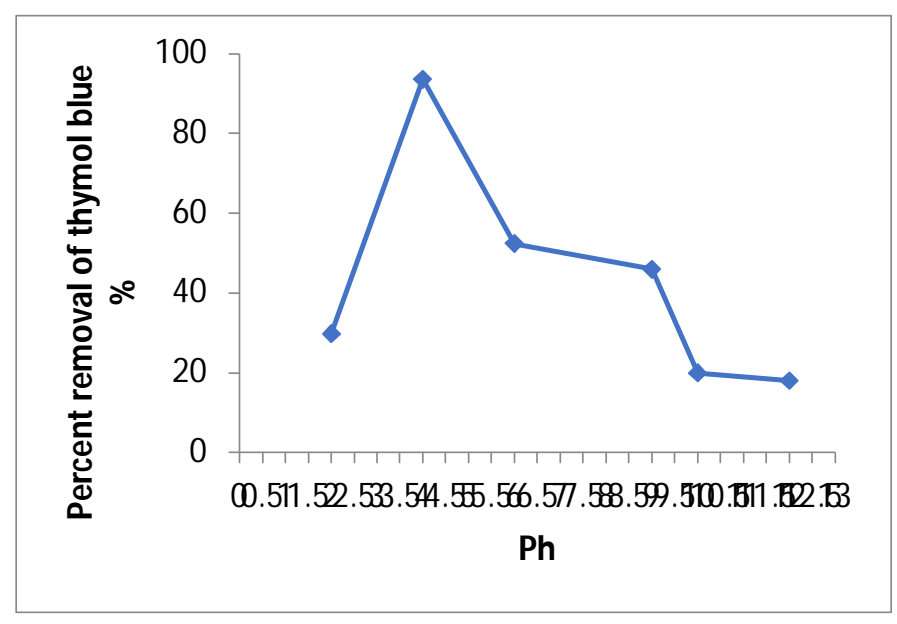

Figure 4: The relation between thymol blue removal percentage and $\mathrm{pH}$

\subsection{Optimum Initial Concentration}

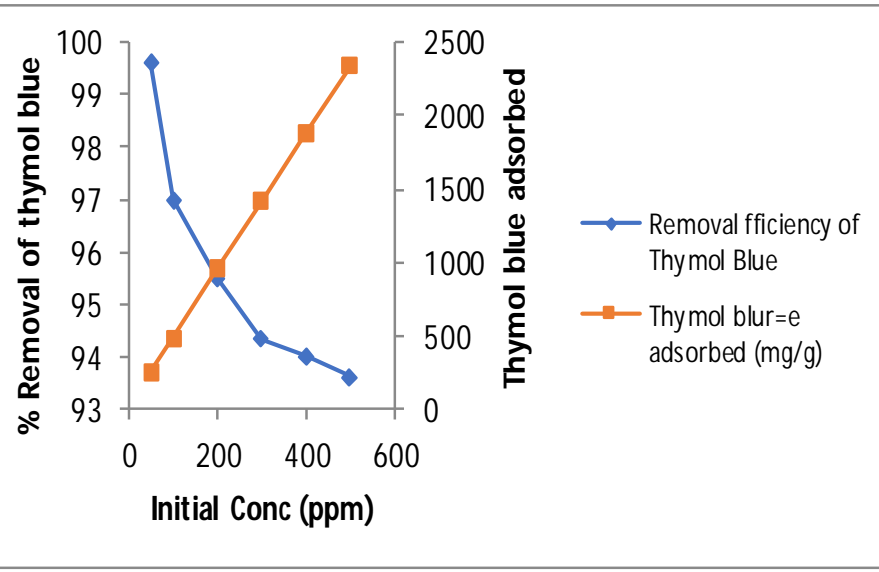

Figure 5: The removal efficiency of thymol blue

The $0.05 \% \mathrm{v} / \mathrm{v}$ sample shows a large value of removal, where the final concentration of thymol blue left in the solution is $0.0002 \% \mathrm{v} / \mathrm{v}$ which means that this sample has lost about $0.498 \% \mathrm{v} / \mathrm{v}$ during adsorption so, by substituting initial and final concentrations in the equation of $\%$ of removal. Percent of removal was $99.6 \%$ which is very high compared to the other concentrations. Also, to calculate thymol blue adsorbed in $\mathrm{mg} / \mathrm{g}$, Thymol blue adsorbed in (ppm) is multiplied by a fraction of volume of solution and mass of adsorbent (Vol. of thymol blue / Mass of chitosan).

\subsection{Optimum Dose of Chitosan}

Figure 6 shows the relation between the dose of chitosan used in the experiment and the final concentration of thymol blue after adsorption, whereas the dose of modified chitosan increase the concentration of thymol blue after adsorption decrease instantly with noticed values which is clear from the points plotted that the least value of final concentration is at the highest amount of modified chitosan used. Where in figure (18) the opposite occur that the percent of removal increase with the amount of modified chitosan used as the final concentration decrease.
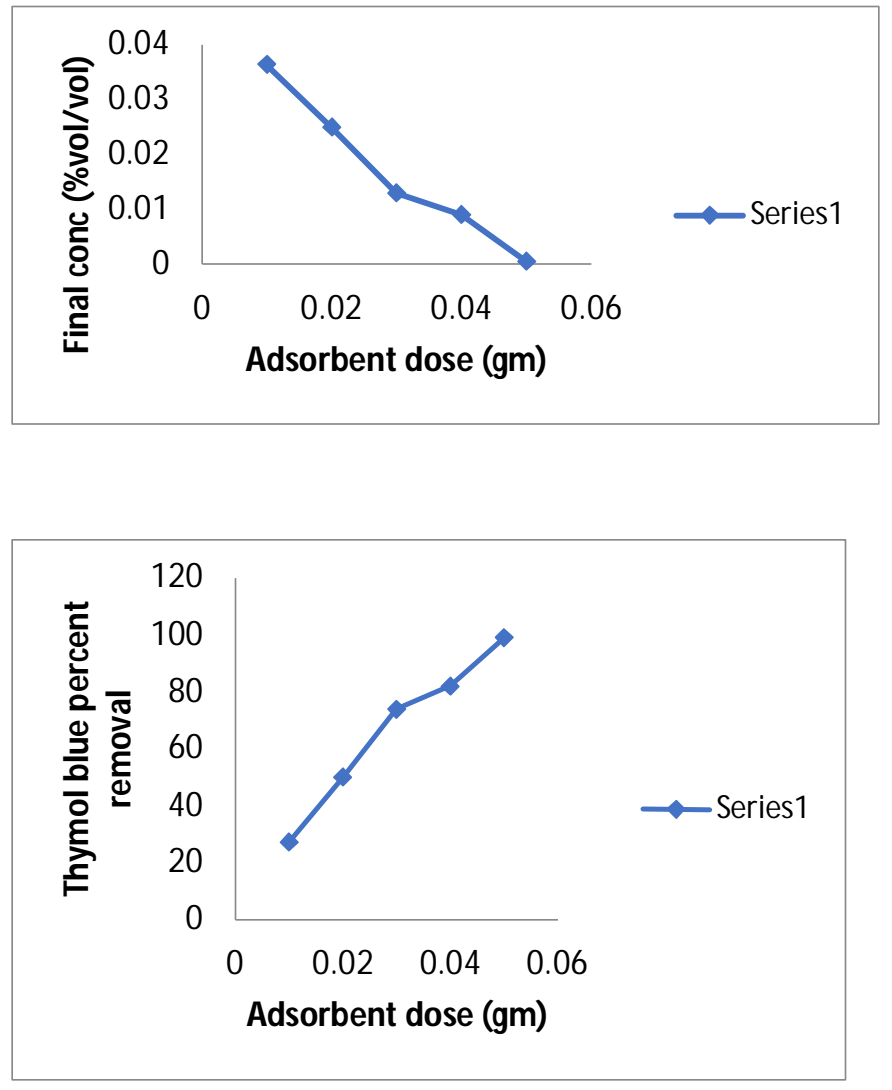

Figure 6 :The relation between dose of chitosan used and final concentration of thymol blue after adsorption

UV/VIS spectrophotometer as shown in figure.7 was used to measure the adsorbance of dye before and after the addition of activated carbon and activated nickel nanoparticles at a wavelength of 665. A UV/VIS spectrophotometer measures the adsorbance of material on an ultraviolet region.

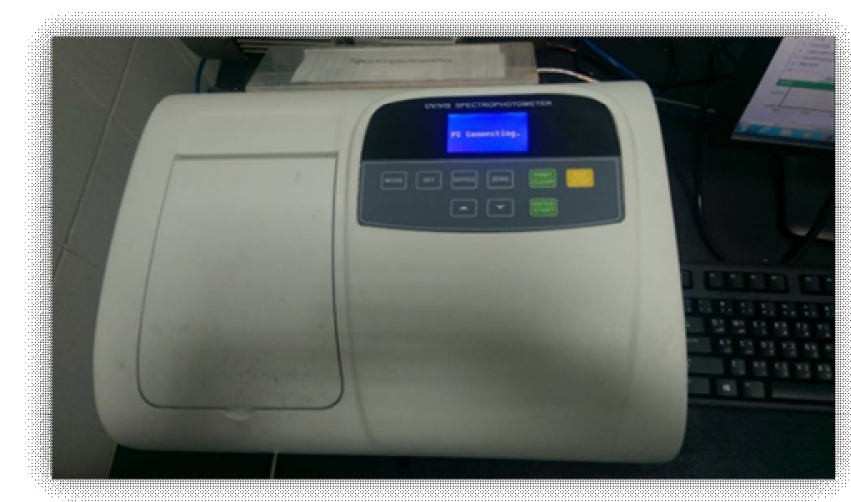

Figure 7:UV-Vis Spectrophotometer 


\subsection{Kinetic Study}

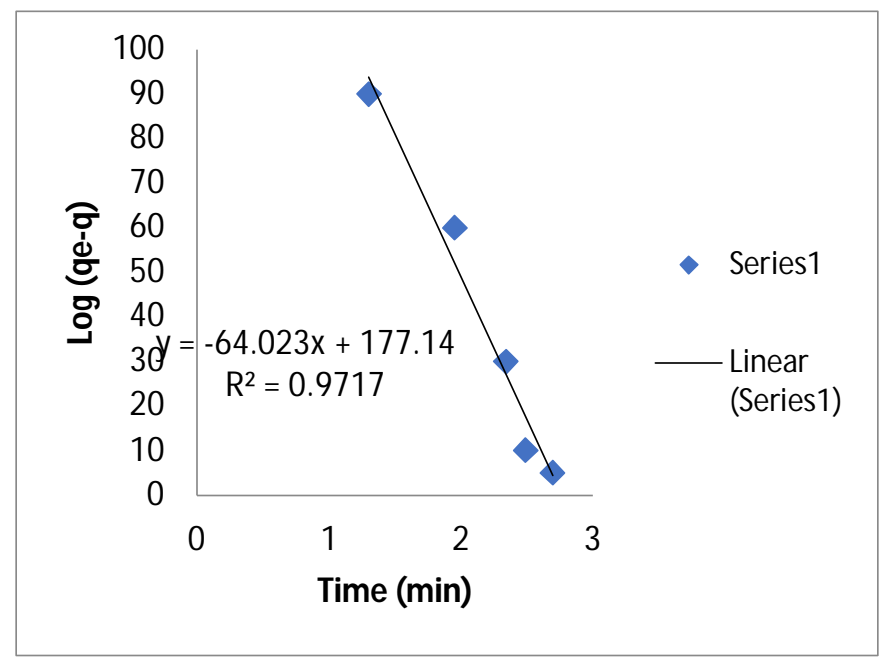

Figure 8: The kinetic model proposed as first order kinetics dye $0.5 \%$

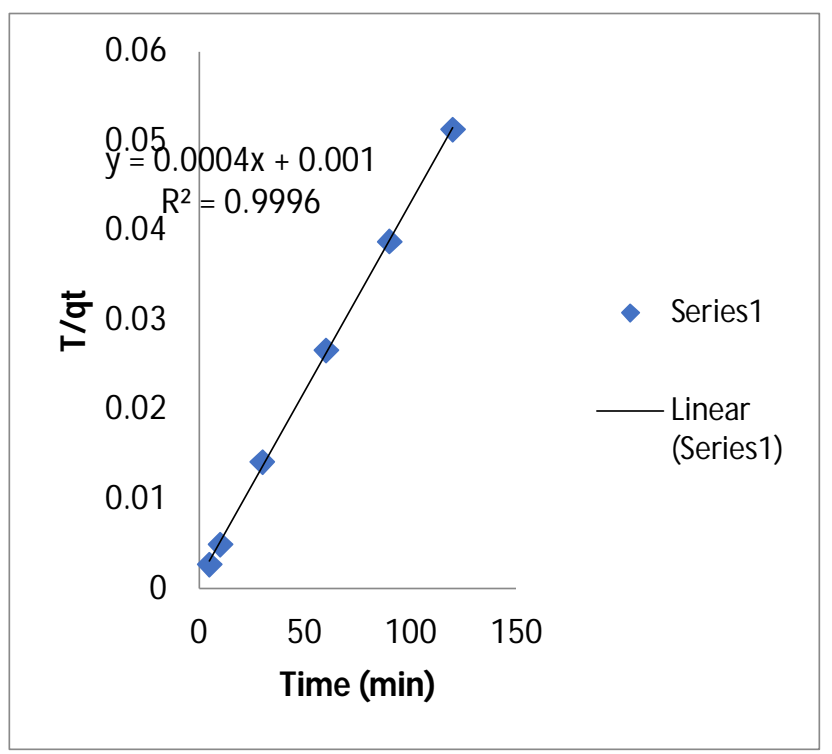

Figure 9: The kinetic model proposed as second order kinetics dye $0.5 \%$

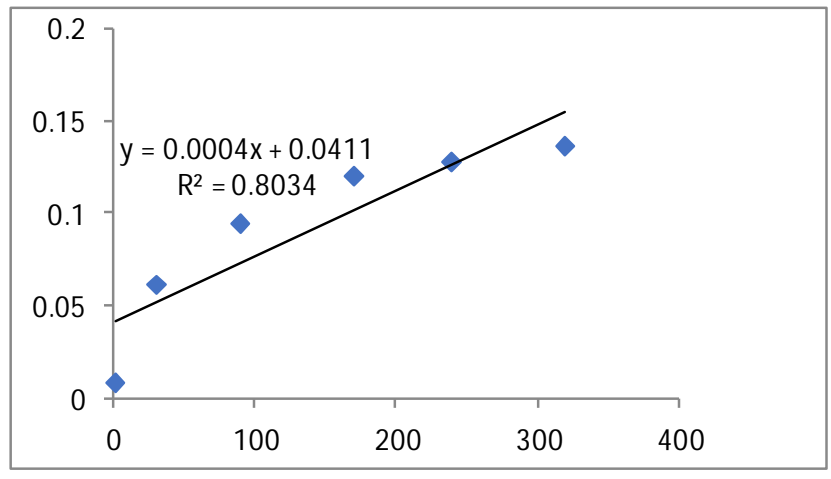

Figure 10: The kinetic model proposed as Langmuir Isotherm
After obtaining the amounts of thymol blue at each concentration it is divided by the mass of the adsorbent which is chitosan having constant value of 0.05 grams to obtain $\left(\mathrm{q}_{\mathrm{e}}\right)$ in $\mathrm{mg} / \mathrm{g}$. From the collected data choosing the best fit is going to be easy by applying some rules and plotting a graph between the logarithmic of $\left(c_{e}\right)$ (x-axis) \& $\left(\mathrm{q}_{\mathrm{e}}\right)$ (y-axis). After plotting the graph, the value of $\mathrm{R}^{2}$ is calculated through the equation given on the graph which is:

$$
\mathrm{Y}=0.0004 \mathrm{x}+0.0411
$$

$\mathrm{R}^{2}=0.8034$

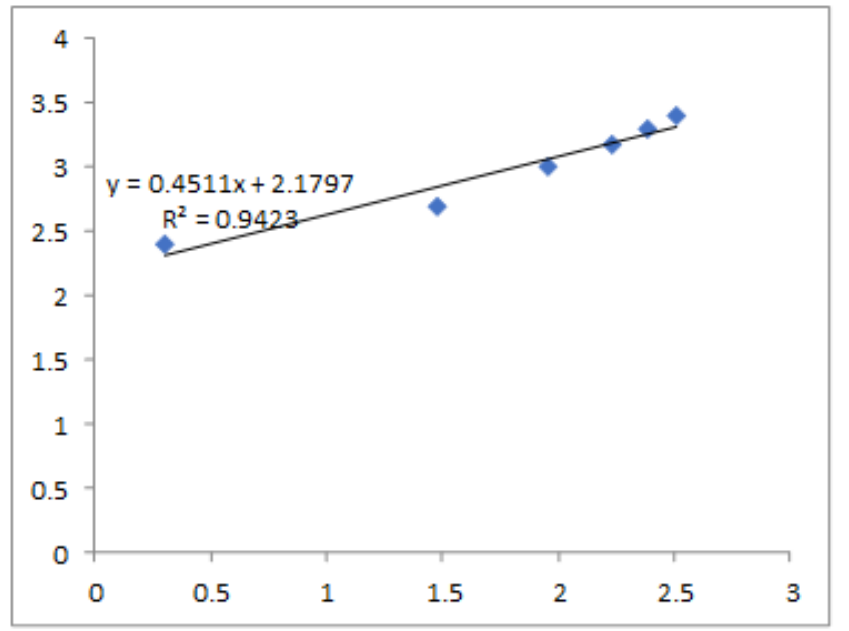

Figure 11: The kinetic model proposed as Frendulish Isotherm

After obtaining the amounts of thymol blue at each concentration it is divided by the mass of the adsorbent which is chitosan having constant value of 0.05 grams to obtain $\left(\mathrm{q}_{\mathrm{e}}\right)$ in $\mathrm{mg} / \mathrm{g}$. From the collected data choosing the best fit is going to be easy by applying some rules and plotting a graph between the logarithmic of $\left(\mathrm{c}_{\mathrm{e}}\right)$ (x-axis) \& $\left(\mathrm{q}_{\mathrm{e}}\right)$ (y-axis). Also, after plotting the graph, the value of $\mathrm{R}^{2}$ is calculated through the equation given on the graph which is $\mathrm{y}=0.4511+2.1797$ $\mathrm{R}^{2}=0.9423$

\section{CONCLUSION}

After preparing the oxidized chitosan, the first experiment which is optimum time took place to obtain the optimum time of adsorption which means to see the interval of time that achieved the best adsorption capacity by decreasing the concentration of thymol blue solution and the least concentration after adsorption process was the best time interval. After lifting each sample when it is time to be removed from the shaker, the samples are then taken to the spectrophotometer to measure the absorbance capacity by measuring the value of concentration of thymol blue after adsorption and a comparison was done between thymol blue before and after adsorption to see the difference in concentration for each sample to observe that the sample that took two hours $(120 \mathrm{~min})$ achieved the best adsorption capacity with absorbance value $0.0035 \mathrm{~nm}$ and concentration 
of thymol blue $0.032 \% \mathrm{v} / \mathrm{v}$. So the percent of removal of oxidized chitosan is $93.6 \%$. While the optimum $\mathrm{pH}$ of the solution was obtained to be 4 after proceeding the optimum time experiment. The spectrophotometer showed that the sample having $\mathrm{pH}$ of 4 achieved the best absorbance value as that of time experiment which is $0.0035 \mathrm{~nm}$ and final concentration of 0.032 with removal percentage $93.6 \%$. Then by concluding the optimum values of time and $\mathrm{pH}$, It was observed that the sample having the concentration $0.05 \% \mathrm{v} / \mathrm{v}$ had the best values of absorbance where the concentration of thymol after adsorption was $0.0002 \% \mathrm{v} / \mathrm{v}$ showing a big difference between the initial and final concentration of thymol blue with percentage of removal $99.6 \%$. The last experiment was the optimum dose of modified chitosan, where after the three previous experiments it was concluded that the optimum values of thymol blue solution was determined. After the process the five samples were taken to the spectrophotometer to conclude that the sample having 0.05 grams oxidized chitosan show a promising adsorption value where the percentage of adsorption was $99 \%$ which means that the remaining thymol blue in solution is negligible by final concentration $0.0005 \% \mathrm{v} / \mathrm{v}$ and $0.014 \mathrm{~nm}$ absorbance value.

Lastly the mathematical relations and equations that was applied on the values collected from the previous experiments was to show whether the reaction is a first or a second order reaction, also knowing the classification of the reaction.

\section{ACKNOWLEDGEMENT}

We express our deep gratitude to British University in Egypt (BUE). This work was partially performed using the facilities at the Nanotechnology Research Centre (NTRC) at the British University in Egypt (BUE).

\section{REFERENCES}

1. Chen S.T., Synthesis of Pd/Fe3O4 Hybrid Nanocatalysts with Controllable Interface and Enhanced Catalytic Activities for CO Oxidation, Journal of Physical Chemistry C, Vol. 116, pp. 2969-12976, March 2012.

https://doi.org/10.1021/jp3036204

2. Radwan N.R.E., El-Shall M.S., Hassan H.M.A., Synthesis and characterization of nanoparticle $\mathrm{Co3O4}, \mathrm{CuO}$ and $\mathrm{NiO}$ catalysts prepared by physical and chemical methods to minimize air pollution, Applied Catalysis A: General, Vol. 331, pp. 8-18, August 2007.

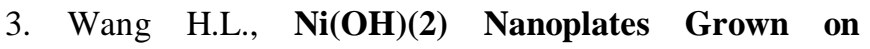
Graphene as Advanced Electrochemical Pseudocapacitor Materials, Journal of the American Chemical Society, Vol. 132, 21, pp. 7472-7477, February 2010.

4. Wang W.W., Zhu Y.J., Ruan M.L., Microwave-assisted synthesis and magnetic property of magnetite and hematite nanoparticles, Journal of Nanoparticle Research, Vol. 9, 3, pp. 419-426, April 2007.

5. Elazab H., Microwave-assisted synthesis of $\mathbf{P d}$ nanoparticles supported on $\mathrm{FeO}, \mathrm{CoO}$, and $\mathrm{Ni}(\mathrm{OH})$ nanoplates and catalysis application for $\mathrm{CO}$ oxidation, Journal of Nanoparticle Research, Vol. 16, 7, pp. 1-11, November 2014.

6. Mankarious R.A., et al., Bulletproof vests/shields prepared from composite material based on strong polyamide fibers and epoxy resin, Journal of Engineering and Applied Sciences, Vol. 12, 10, pp. 2697-2701, May 2017.

7. Mohsen W., Sadek M.A., Elazab H.A., Green synthesis of copper oxide nanoparticles in aqueous medium as a potential efficient catalyst for catalysis applications, International Journal of Applied Engineering Research, Vol. 12, 24, pp. 14927-14930, July 2017.

8. Mostafa A.R., Omar H.A.-S., Hany A.E., Preparation of Hydrogel Based on Acryl Amide and Investigation of Different Factors Affecting Rate and Amount of Absorbed Water, Agricultural Sciences, Vol. 8, pp. 2-11, June 2017.

9. Radwan M.A., et al., Mechanical characteristics for different composite materials based on commercial epoxy resins and different fillers, Journal of Engineering and Applied Sciences, Vol. 12, 5, pp. 1179-1185, May 2017.

10. Andrade A.L., Catalytic Effect of Magnetic Nanoparticles Over the $\mathbf{H}(2) \mathrm{O}(2)$ Decomposition Reaction, Journal of Nanoscience and Nanotechnology, Vol. 9, 6, pp. 3695-3699, June 2009.

https://doi.org/10.1166/jnn.2009.NS53

11. Kustov A.L., CO methanation over supported bimetallic Ni-Fe catalysts: From computational studies towards catalyst optimization, Applied Catalysis a-General, Vol. 320, pp. 98-104, November 2007.

12. Lohitharn N., Goodwin J.G., Impact of $\mathbf{C r}, \mathbf{M n}$ and $\mathbf{Z r}$ addition on Fe Fischer-Tropsch synthesis catalysis: Investigation at the active site level using SSITKA, Journal of Catalysis, Vol. 257, 1, pp. 142-151, August 2008.

13. Moreau F., Bond G.C., CO oxidation activity of gold catalysts supported on various oxides and their improvement by inclusion of an iron component, Catalysis Today, Vol. 114, 4, pp. 362-368, October 2006.

14. Sarkari M., Fischer-Tropsch synthesis: Development of kinetic expression for a sol-gel $\mathrm{Fe}-\mathrm{Ni} / \mathrm{Al2O3}$ catalyst, Fuel Processing Technology, Vol. 97, pp. 130-139, April 2012.

15. Elazab H., The Effect of Graphene on Catalytic Performance of Palladium Nanoparticles Decorated with $\mathrm{FeO}$, $\mathrm{CoO}$, and Ni (OH): Potential Efficient Catalysts Used for Suzuki Cross-Coupling, Catalysis Letters, Vol. 147, 6, pp. 1510-1522, July 2017.

16. Elazab H.A., The continuous synthesis of Pd supported on $\mathrm{Fe} 3 \mathrm{O} 4$ nanoparticles: A highly effective and 
magnetic catalyst for CO oxidation, Green Processing and Synthesis, Vol. 6, 4, pp. 413-424, May 2017.

17. Elazab H.A., Sadek M.A., El-Idreesy T.T., Microwave-assisted synthesis of palladium nanoparticles supported on copper oxide in aqueous medium as an efficient catalyst for Suzuki cross-coupling reaction, Adsorption Science \& Technology, Vol. 36, pp. 1352-1365, May 2018.

18. Elazab H.A., Highly efficient and magnetically recyclable graphene-supported $\mathrm{Pd} / \mathrm{Fe} 304$ nanoparticle catalysts for Suzuki and Heck cross-coupling reactions, Applied Catalysis A: General, Vol. 491, pp. 58-69, February 2015.

19. Hirvi J.T., et al., CO oxidation on PdO surfaces, Journal of Chemical Physics, Vol. 133, pp. 8-14, August 2010.

20. Iglesias-Juez A., Nanoparticulate Pd Supported Catalysts: Size-Dependent Formation of $\operatorname{Pd}(\mathrm{I}) / \operatorname{Pd}(0)$ and Their Role in CO Elimination, Journal of the American Chemical Society, Vol. 133, 12, pp. 4484-4489, March 2011.

21. Ivanova A.S., Metal-support interactions in Pt/Al2O3 and Pd/Al2O3 catalysts for $\mathrm{CO}$ oxidation, Applied Catalysis B-Environmental, Vol. 97, 1-2, pp. 57-71, April 2010.

22. Kim H.Y., Henkelman G., CO Oxidation at the Interface between Doped $\mathrm{CeO} 2$ and Supported Au Nanoclusters, Journal of Physical Chemistry Letters, Vol. 3, 16, pp. 2194-2199, January 2012.

23. Chattopadhyay K., Dey R., Ranu B.C., Shape-dependent catalytic activity of copper oxide-supported Pd(0) nanoparticles for Suzuki and cyanation reactions, Tetrahedron Letters: International Organ for the Rapid Publication of Preliminary Communications in Organic Chemistry, Vol. 50, 26. pp. 3164-3167, December 2009.

24. Hoseini S.J., Modification of palladium-copper thin film by reduced graphene oxide or platinum as catalyst for Suzuki-Miyaura reactions, Applied Organometallic Chemistry, Vol. 31, pp. 5-12, March 2017.

https://doi.org/10.1002/aoc.3607

25. Hosseini-Sarvari M., Razmi Z., Palladium Supported on Zinc Oxide Nanoparticles as Efficient Heterogeneous Catalyst for Suzuki Miyaura and Hiyama Reactions under Normal Laboratory Conditions, Helvetica Chimica Acta, Vol. 98, 6, pp. 805-818, April 2015.

26. Nasrollahzadeh M., Ehsani A., Jaleh B., Preparation of carbon supported CuPd nanoparticles as novel heterogeneous catalysts for the reduction of nitroarenes and the phosphine-free Suzuki Miyaura coupling reaction, New Journal of Chemistry, Vol. 39, 2, pp. 1148-1153, February 2015.

27. Nasrollahzadeh M., Palladium nanoparticles supported on copper oxide as an efficient and recyclable catalyst for carbon(sp2) carbon(sp2) cross-coupling reaction, Materials Research Bulletin, Vol. 68, pp. 150-154, April 2013.

28. Mandali P.K., Chand D.K., Palladium nanoparticles catalyzed Suzuki cross-coupling reactions in ambient conditions, Catalysis Communications, Vol. 31, pp.16-20, November 2016.

29. Wang Y., CuO Nanorods-Decorated Reduced Graphene Oxide Nanocatalysts for Catalytic Oxidation of CO, Catalysts, Vol. 6, 12, pp. 214-220, September 2016.

30. Igarashi H., Uchida H., Watanabe M., Mordenite-supported noble metal catalysts for selective oxidation of carbon monoxide in a reformed gas, Chemistry Letters, Vol. 11, pp. 1262-1263, October 2000.

31. Liu W.H., Fleming S., Lairson B.M., Reduced intergranular magnetic coupling in $\mathrm{Pd} / \mathrm{Co}$ multilayers, Journal of Applied Physics, Vol. 79, 7, pp. 3651-3655, May 1996. https://doi.org/10.1063/1.361193

32. Luo J.Y., Mesoporous $\operatorname{Co}(3) \mathrm{O}(4)-\mathrm{CeO}(2)$ and $\mathrm{Pd} / \mathrm{Co}(3) \mathrm{O}(4)-\mathrm{CeO}(2) \quad$ catalysts: Synthesis, characterization and mechanistic study of their catalytic properties for low-temperature CO oxidation, Journal of Catalysis, Vol. 254, 2, pp. 310-324, June 2008.

33. Pavlova S.N., The influence of support on the low-temperature activity of $\mathrm{Pd}$ in the reaction of $\mathrm{CO}$ oxidation on Kinetics and mechanism of the reaction, Journal of Catalysis, Vol. 161, 2, pp. 517-523, May 1996.

34. Diyarbakir S.M., Can H., Metin Ã.n., Reduced Graphene Oxide-Supported CuPd Alloy Nanoparticles as Efficient Catalysts for the Sonogashira Cross-Coupling Reactions, Acs Applied Materials \& Interfaces, Vol. 7, 5, pp. 3199-3206, March 2015.

35. Feng Y.-S., et al., ChemInform Abstract: $\mathbf{P d C u}$ Nanoparticles Supported on Graphene: An Efficient and Recyclable Catalyst for Reduction of Nitroarenes, ChemInform, Vol. 46, pp. 4-12, August 2015.

36. Feng Y.-S., et al., PdCu nanoparticles supported on graphene: an efficient and recyclable catalyst for reduction of nitroarenes, Tetrahedron, Vol. 70, 36, pp. 6100-6105, May 2014.

37. Liu Y., et al., Ultrasensitive electrochemical immunosensor for SCCA detection based on ternary $\mathbf{P t} / \mathbf{P d C u}$ nanocube anchored on three-dimensional graphene framework for signal amplification, Biosensors \& Bioelectronics, Vol. 79, pp. 71-78, July 2016.

38. Shafaei Douk A., Saravani H., Noroozifar M., Novel fabrication of $\mathrm{PdCu}$ nanostructures decorated on graphene as excellent electrocatalyst toward ethanol oxidation, International Journal of Hydrogen Energy, Vol. 42, 22, pp. 15149-15159, August 2017. 
39. Hany A. Elazab, Investigation of Microwave-assisted Synthesis of Palladium Nanoparticles Supported on $\mathrm{Fe}_{3} \mathrm{O}_{4}$ as an Efficient Recyclable Magnetic Catalysts for Suzuki Cross - Coupling, The Canadian Journal of Chemical Engineering, Vol. 96, 12, pp. 250-261, January 2019.

40. Hany A. Elazab, Laser Vaporization and Controlled Condensation (LVCC) of Graphene supported $\mathrm{Pd} / \mathrm{Fe}_{3} \mathrm{O}_{4}$ Nanoparticles as an Efficient Magnetic Catalysts for Suzuki Cross - Coupling, Biointerface Research in Applied Chemistry, Vol. 8, 3, pp. 3314 3318, August 2018.

41. Hany A. Elazab, The catalytic Activity of Copper Oxide Nanoparticles towards Carbon Monoxide Oxidation Catalysis: Microwave - Assisted Synthesis Approach, Biointerface Research in Applied Chemistry, Vol. 8, 3, pp. 3278 - 3281, June 2018.

42. M. A. Radwan, Omar Al-Sweasy, M. A. Sadek, Hany A. Elazab, Investigating the Agricultural Applications of Acryl Amide based Hydrogel, International Journal of Engineering and Technology(UAE), Vol. 7, 4.29, pp. 168-171, April 2018.

43. Fatma Zakaria, M. A. Radwan, M. A. Sadek, Hany A. Elazab, Insulating material based on shredded used tires and inexpensive polymers for different roofs, International Journal of Engineering and Technology(UAE), Vol. 7, 4, pp. 1983-1988, June 2018.

44. Reem Nasser, M. A. Radwan, M. A. Sadek, Hany A. Elazab, Preparation of insulating material based on rice straw and inexpensive polymers for different roofs, International Journal of Engineering and Technology(UAE), Vol. 7, 4, pp. 1989-1994, June 2018.

45. Mostafa Ghobashy, Mamdouh Gadallah, Tamer $\mathrm{T}$. El-Idreesy, M. A. Sadek, Hany A. Elazab, Kinetic Study of Hydrolysis of Ethyl Acetate using Caustic Soda, International Journal of Engineering and Technology(UAE), Vol. 7, 4, pp. 1995-1999, June 2018.

46. Nourhan Sherif Samir, Mostafa A. Radwan, M. A. Sadek, Hany A. Elazab, Preparation and Characterization of Bullet-Proof Vests Based on Polyamide Fibers, International Journal of Engineering and Technology(UAE), Vol. 7, 3, pp. 1290-1294, May 2018.

47. Basant Ashraf, Mostafa A. Radwan, M. A. Sadek, Hany A. Elazab, Preparation and Characterization of Decorative and Heat Insulating Floor Tiles for Buildings Roofs, International Journal of Engineering and Technology (UAE), Vol. 7, 3, pp. 1295-1298, May 2018.

48. Mandali P.K., Chand D.K., Palladium nanoparticles catalyzed Suzuki cross-coupling reactions in ambient conditions, Catalysis Communications, Vol. 31, 5, pp. 16-20, October 2016.

49. Wang Y., CuO Nanorods-Decorated Reduced Graphene Oxide Nanocatalysts for Catalytic Oxidation of CO, Catalysts, Vol. 6, 12, pp. 214-223, April 2016.
50. Pavlova S.N., The influence of support on the low-temperature activity of $\mathrm{Pd}$ in the reaction of $\mathrm{CO}$ oxidation on Kinetics and mechanism of the reaction, Journal of Catalysis, Vol. 161, 2, pp. 517-523, July 1996.

51. Diyarbakir S.M., Can H., Metin Ã.n., Reduced Graphene Oxide-Supported CuPd Alloy Nanoparticles as Efficient Catalysts for the Sonogashira Cross-Coupling Reactions, Acs Applied Materials \& Interfaces, Vol. 7, 5, pp. 3199-3206, June 2015.

52. M. A. Radwan, Mohamed Adel Rashad, M. A. Sadek, Hany A. Elazab, Synthesis, Characterization and Selected Application of Chitosan Coated Magnetic Iron Oxide Nanoparticles, Journal of Chemical Technology and Metallurgy, Vol. 54, 2, pp. 303-310, June 2019.

53. Hosam H. Abdelhady, Hany A. Elazab, Emad M. Ewais, Mohamed Saber, Mohamed S. El-Deab, Efficient Catalytic Production of Biodiesel Using Nano-Sized Sugarbeet Agro-Industrial waste, Fuel, Vol. 261, pp. 116481, February 2020. https://doi.org/10.1016/j.fuel.2019.116481

54. Hany A. Elazab, M. A. Sadek, Tamer T. El-Idreesy, Facile Synthesis of Reduced Graphene Oxide-Supported $\mathrm{Pd} / \mathrm{CuO}$ Nanoparticles as an Efficient Catalyst for Cross-Coupling Reactions, Journal of Chemical Technology and Metallurgy, Vol. 54, 5, pp. 934-946, August 2019.

55. Hany A. Elazab, Tamer T. El-Idreesy, Polyvinylpyrrolidone - Reduced Graphene Oxide - Pd Nanoparticles as an Efficient Nanocomposite for Catalysis Applications in Cross-Coupling Reactions, Bulletin of Chemical Reaction Engineering and Catalysis, Vol. 14, 3, pp. 490-501, December 2019.

56. Hany A. Elazab, Ali R. Siamaki, B. Frank Gupton, M. Samy El-Shall, $\mathbf{P d}-\mathrm{Fe}_{3} \mathrm{O}_{4} / \mathbf{R G O}$ : a Highly Active and Magnetically Recyclable Catalyst for Suzuki Cross Coupling Reaction using a Microfluidic Flow Reactor, Bulletin of Chemical Reaction Engineering and Catalysis, Vol. 14, 3, pp. 478-489, December 2019.

57. Hany A. Elazab, M. A. Radwan, Tamer T. El-Idreesy, Facile microwave-assisted synthetic approach to palladium nanoparticles supported on copper oxide as an efficient catalyst for Heck cross-coupling reactions, International Journal of Nanoscience, Vol. 18, 5, pp. 1850032, June 2019.

58. Hany A. Elazab, S. A. Hassan, M. A. Radwan, M. A. Sadek, Microwave-assisted Synthesis of Graphene supported Hexagonal Magnetite for Applications in Catalysis, International Journal of Innovative Technology and Exploring Engineering (IJITEE), Vol. 8, 12, 5511-5513, 2019.

59. Hany A. Elazab, M. A. Radwan, M. A. Sadek, Hydrothermal Synthesis of Palladium nanoparticles supported on $\mathrm{Fe}_{3} \mathrm{O}_{4}$ Nanoparticles: an Efficient Magnetic Catalysts for CO Oxidation, International Journal of Innovative Technology and Exploring 
Engineering (IJITEE), Vol. 8, 12, pp. 2792-2794, May 2019.

60. Tarek M. Aboul-Fotouh, Sherif K. Ibrahim, M. A. Sadek, Hany A. Elazab, High Octane Number Gasoline-Ether Blend, International Journal of Innovative Technology and Exploring Engineering (IJITEE), Vol. 8, 9, pp. 732-739, March 2019.

61. Tarek M. Aboul-Fotouh, Islam Alaa, M. A. Sadek, Hany A. Elazab, Physico-Chemical Characteristics of Ethanol-Diesel Blend Fuel, International Journal of Innovative Technology and Exploring Engineering (IJITEE), Vol. 8, 9, pp. 740-747, February 2019.

62. Hany A. Elazab, M. M. Seleet, Said M. A. Hassanein, M. A. Radwan, M. A. Sadek, Synthesis and Characterization of Dinitro Pentamethylene Tetramine (DPT), Journal of Advanced Research in Dynamical and Control System, Vol. 11, 5S, pp. 310-318, August 2019.

63. Hany A. Elazab, M. M. Seleet, Said M. A. Hassanein, M. A. Radwan, M. A. Sadek, Follow-up and Kinetic Model Selection of Dinitro Pentamethylene Tetramine (DPT), International Journal of Innovative Technology and Exploring Engineering (IJITEE), Vol. 8, 8, pp. 2862-2866, October 2019.

64. Hany A. Elazab, Mamdouh Gadall, M. A. Sadek, Tamer T. El-Idreesy, Hydrothermal Synthesis of Graphene supported $\mathrm{Pd} / \mathrm{Fe}_{3} \mathrm{O}_{4}$ Nanoparticles as an Efficient Magnetic Catalysts for Suzuki Cross - Coupling, Biointerface Research in Applied Chemistry, Vol. 9, 2, pp. 3906-3911, March 2019.

65. Hany A. Elazab, M. M. Seleet, Said M. A. Hassanein, M.
A.
Radwan,
M.
A. Sadek, 3,7-Dinitro-1,3,5,7-Tetraazabicyclo[3,3,1]Nonane (DPT): An Important Intermediate in the Synthesis Route of one of the Most Powerful Energetic Materials (RDX/HMX), International Journal of Innovative Technology and Exploring Engineering (IJITEE), Vol. 8, 452, pp. 88-95, April 2019.

66. Hany A. Elazab, Tamer T. El-Idreesy, Optimization of the Catalytic Performance of $\mathrm{Pd} / \mathrm{Fe}_{3} \mathrm{O}_{4}$ Nanoparticles Prepared via Microwave-assisted Synthesis for Pharmaceutical and Catalysis Applications, Biointerface Research in Applied Chemistry, Vol. 9, 1, pp. 3794-3799, July 2019.

https://doi.org/10.33263/BRIAC91.794799 